\title{
Comments on "Origin of Abnormality in a Human Simelian Foetus as Elucidated by Our Knowledge of Vertebrate Development"
}

\author{
To the Editor:
}

In their recent paper on the origin of abnormality in a human simelian foetus Chandebois and Brunet ('87) state that "the defects concerned both cephalic and abdominal regions." From the photographs of the fetus and the limited description in the text, they have not made a case for true malformation of the cephalic region. What they have shown is oligohydramniotic (compressive) deformation. This type of craniofacial alteration is regularly seen when there has been severe, prolonged oligohydramnios of any etiology, be it renal agenesis, urethral or ureteral obstruction, or amniorrhea. Thus, their speculations on excessive length of prechordal mesoderm initiating cephalic maldevelopment are irrelevant.

Chandebois and Brunet use the word fusion to depict the development of the symmelia. Fusion implies the apposition of two processes that join following the breakdown of their epithelial investments, as seen in the fusion of the maxillary and frontonasal processes of the face. A more appropriate term for what happens in the genesis of symmelia is merging of the anlagen of the posterior limb buds along their postaxial margins. Merging implies that the separation of two processes is obliterated by the filling-in of an intervening sulcus, without epithelial breakdown. This is the process involved in formation of the lower lip and of the tongue; in the case of symmelia, the absence of tissue normally interposed between the prospective limb buds allows those regions to merge with each other across the midline.

If one reviews the many descriptions of simelian fetuses in the literature, it is apparent that there are exceptions to the dogma that simelians have agenesis of the urinary tract, hindgut, and caudal vertebrae. For example, Monie ('74) reported a case with kidneys, Newbill ('41) reported a case with a patent tract from the external urethral meatus to the umbilical ring, Hilbelink et al. ('75) described a fetus with a normal gut including a patent anus, and $I$ have seen two fetuses with intact but dorsally displaced sacrococcygeal vertebrae (unpublished observations).

The variable presence of renal tissue can be accounted for less mysteriously than Chandebois and Brunet suggest. Kidneys are developed from the intermediate mesoderm and are dependent on the prior formation of the mesonephroi and mesonephric ducts, which originate well cephalad to the caudal region. In a developing siren the mesonephric ducts are presumed to extend for a variable distance into the caudal end of the embryo; that the mesonephroi and their ducts usually do develop is evident from the finding of normal epididymus and proximal vas deferens in sirens (male). If the mesonephric ducts extend far enough to give rise to the ureteric buds, these buds will enter the metanephric blastema and induce kidney formation, even though no bladder may be present. However, the metanephric portion of the intermediate mesoderm may also be damaged/defective (from its proximity to damaged/defective paraxial mesoderm) and thus form hypoplastic or otherwise abnormal kidneys. Should the mesonephric ducts be unable to penetrate the caudal region far enough to give rise to the ureteric buds or if the metanephric blastema is also destroyed, no kidney will be formed.

From consideration of the reported cases of symmelia, from my own dissections, and from review of the literature on animal models of "caudal dysplasia," it seems to me that the primary insult is to a focus of tissue in the caudal end of the embryo during the third or fourth week. The nature of the insult may be variable but a hematoma is an appropriate conceptual model. The defects that result are a function of the spatial extent of the damage/defect, varying along ventro-dorsal, ce- 
phalo-caudal, and left-right axes. There is further variation according to the time and duration of the insult. I believe that this concept helps explain the variability in anatomic detail among sirens and does not exclude the possibility of true malformations in parts or systems remote from the caudal end of the embryo. The speculations advanced by Chandebois and Brunet apply in part to the single case they present but do not suffice to describe all simelian fetuses.

\section{LITERATURE CITED}

Chandebois, R., and C. Brunet (1987) Origin of abnormality in a human simelian foetus as elucidated by our knowledge of vertebrate development. Teratology, $36: 11-22$

Hilbelink, D.R., S.Y. Long, and S. Kaplan (1975) Caudal dysplasia syndrome (sirenomelia in man; rumplessness in the chick): A common etiology? Teratology, $11: 22 \mathrm{~A}$.

Monie, I.W. (1974) Human symmelia: Determination of sex and other features. Teratology, 9:87-92.

Newbill, H.P. (1941) Sirenomelian monster (sympus): An anatomic presentation. Am. J. Dis. Child., 62:12331241.

MASON BARR, JR. Departments of Pediatrics and Pathology University of Michigan

Ann Arbor, MI 48109 